Respuestas

Año 17

No. 1

Junio 2012

ISSN 0122-820X

PP: 66-77

\section{La innovación tecnológica en las empresas del sector cerámico del área metropolitana de Cúcuta}

Patricia Ramírez Delgado ${ }^{1}$

\section{Resumen}

Recibido:

Noviembre 2 de 2011

Aceptado:

Abril 26 de 2012
Con la investigación se busca realizar una caracterización del proceso de innovación tecnológica en las empresas cerámicas del área metropolitana de Cúcuta. La metodología utilizada consistió en la aplicación de una encuesta estructurada, a los gerentes o jefes de producción de las 37 empresas que participaron en el estudio. El instrumento se diseñó para medir las capacidades y el proceso de innovación, partiendo de los diferentes aportes realizados por los expertos en el tema, y los Manuales de Oslo y de Bogotá. Del análisis de los resultados se evidenció que el sector cerámico no cuenta con las capacidades de capital humano, organización, exploración y explotación que favorezcan los procesos de innovación tecnológica; los procesos de innovación actuales se caracterizan por ser adaptativos e incrementales de los desarrollos exógenos; y existe una débil articulación de las empresas del sector cerámico con el Sistema Nacional de Ciencia, Tecnología e Innovación. La investigación proporciona un instrumento que sirve para caracterizar la innovación tecnológica y analizarla por medio de perfiles, que puede ser adaptado a cualquier empresa del sector manufacturero.

Palabras clave: innovación tecnológica, capacidad de innovación, proceso de innovación.

\section{Abstract}

With research is to realize a process characterization technological innovation in ceramic companies in metropolitan Cucuta. The Methodology consisted of a survey structured, managers or heads of production of the 37 companies that participated in the study. The instrument was designed to measure the skills and the innovation process, based on the different contributions made by the experts, and Oslo manuals and Bogota. Analysis of the results showed that the ceramic sector does not have the human capital capabilities, organization, exploration and exploitation which foster technological innovation; innovation processes current characterized by adaptive and incremental developments
${ }^{1}$ Grupo de Investigación en Tecnología Cerámica, Universidad Francisco de Paula

Santander, Colombia.

Magíster en Gerencia de Empresas Ing.patriciaramirez.d@gmail.com 
exogenous and there is a weak link in the ceramic sector companies with System National Science, Technology and Innovation. The research provides an instrument used to characterize and technological innovation analyze it by means of profiles, which can be adapted to any company the manufacturing sector.

Keywords: Technological innovation, innovation, process innovation.

\section{Introducción}

$\mathrm{D}$ entro de los primeros expositores de la innovación se encuentra Shumpeter (1939 citado en Mandonado, Fernández y Doiro, 2003), quien manifiesta que "la innovación es el establecimiento de una función de producción nueva, es decir, un nuevo producto, una nueva forma de organización como por ejemplo la fusión y apertura de mercados nuevos".

El término innovación se ha convertido en un factor clave del desarrollo económico, tecnológico y social de los países. En efecto, la innovación no pasa desapercibida dentro de los sistemas nacionales, y por ello se habla de innovación a cinco niveles (Pineda, 2008):

- A nivel macroeconómico la innovación se refiere a las políticas y estrategias de Ciencia y Tecnología e Investigación y Desarrollo que adoptan los países para generar un entorno favorable a la innovación.

- A nivel mesoeconómico, se refiere a la institucionalidad de la innovación que en muchos casos se refleja en los llamados Sistemas Nacionales de Innovación y/o Sistemas Regionales de Innovación.

- A nivel microeconómico, se refiere a las empresas como los principales dinamizadores del proceso de innovación en PPSMN: productos, procesos, servicios y modelos de negocios.
- A nivel de las innovaciones organizacionales es la necesidad de la gestión de cambio dentro de las organizaciones para poderlas adaptar a los nuevos entornos de competitividad.

- A nivel de las innovaciones sociales, lo cual busca todo proceso de innovación que es conducir a mejoras de la calidad de vida. La innovación en cualquiera de los niveles anteriormente mencionados, debe buscar mejoras en la productividad, lo cual deberá conducir a que se trabaje menos y se tengan mayores ingresos, o a que se trabaje más pero también que el nivel de remuneración se vea reflejada en su capacidad de ahorro, y mejoras en la calidad de vida de la población.

La innovación hace referencia a todo cambio, basado en conocimiento de cualquier tipo, siempre que genera valor $y$ cuando tenga consecuencias económicas directas. Evidentemente, entre ellas están las innovaciones tecnológicas, que son las que generan más beneficios porque no solamente son causa de mejores prestaciones en la oferta de la empresa sino que son más difícilmente imitables y por lo tanto se mantienen en el mercado mayor tiempo como novedad.

La innovación juega un papel importante en la competitividad de los sectores, tal como lo expone Porter (1990): "La competitividad de una nación depende de la capacidad de su industria para innovar y mejorar. La empresa 
consigue ventaja competitiva mediante innovaciones".

El OCDE (2005), sostiene que la innovación es la implantación de un producto, proceso o servicio nuevo o significativamente mejorado. Así mismo, la innovación será un nuevo método de comercialización, un nuevo método de organización para la práctica del negocio o una nueva forma de relaciones externas.

Según el criterio del OCDE (2005), las actividades que permiten llevar el conocimiento tecnológico al mercado se clasifican en tres grupos:

El primero, "actividades de generación y adquisición de conocimiento" formado por la $\mathrm{I}+\mathrm{D}$ realizada o financiada por la empresa y la compra de inmovilizado material e inmaterial (licencias, patentes, Know-how, etc.).

El segundo, "preparación para la producción", donde se incluyen el diseño e ingeniería del producto, la ingeniería de proceso y las llamadas pre-series de producción.

El tercero, "preparación para la comercialización", modelos de negocio donde se logre una evidente generación de valor.

Se puede considerar como innovación a todo cambio que genera valor para la empresa. Si los nuevos productos, procesos o servicios no son aceptados por el mercado, no existe innovación.

Diversas metodologías aceptadas internacionalmente, constituyen las referencias clásicas en la tipificación de conceptos y medición de los insumos y los resultados económicos y tecnológicos de la $I \& D$ y la innovación. Entre ellas encuentran el Manual de Frascati, el Manual de Oslo y el Manual de Bogotá. Según en el Manual de Oslo de la OCDE las actividades de innovación tecnológica de producto-proceso (TPP), la define como todos aquellos pasos científicos, organizacionales, financieros y comerciales orientados a la implementación de productos o procesos nuevos o tecnológicamente mejorados. Algunos pueden ser propiamente innovativos en sí mismos, mientras que otros pueden no ser novedosos, pero si necesarios para la implementación. Según el Manual de Bogotá, las innovaciones según su tipo, "comprenden los productos y procesos implementados tecnológicamente nuevos, como también las mejoras tecnológicas de importancia producidas en productos y procesos. Se considera que una innovación TPP ha sido implementada si se la introdujo en el mercado (innovación de producto) o si se la usó dentro de un proceso de producción (innovación de proceso)". En la presente investigación sólo se consideran las innovaciones de producto y proceso.

Según el grado de novedad la innovación se puede clasificar en (Imai, 1989):

Innovación radical: tiene su origen en una teoría científica o en un avance tecnológico cuantitativamente importante. Para conseguir esta innovación normalmente se requiere de personal científico muy cualificado.

Innovación incremental: consiste en realizar mejoras sobre la tecnología existente, que modifican sustancialmente la capacidad competitiva de la empresa a largo plazo, pero permite conseguir importantes ahorros en costos, así como mejoras funcionales en productos.

Innovación imitativa: es un caso de innovación incremental, cuyo objetivo es copiar la esencia de una tecnología de la competencia, para mejorarla después funcionalmente.

En la elaboración de la caracterización de la innovación tecnológica de las empresas del sector cerámico del área metropolitana de Cúcuta, se tuvo en cuenta medir la capacidad de innovación y el proceso de innovación, bajo lo expuesto por los diferentes enfoques que se presentan a continuación. 
Los factores determinantes de la capacidad innovadora comprenden aspectos tanto de carácter interno como externo. Dentro de los determinantes internos se encuentran:

El carácter innovador de las empresas viene determinado, en gran medida, por los factores internos de las mismas (Hadjimanolis, 2000). Estos factores se basan en los recursos, capacidades y competencias, que permiten a las empresas diferenciarse a través de ventajas competitivas sostenibles, basadas en la singularidad, la complementariedad de los recursos y la dificultad para ser copiadas.

Al considerar los factores internos, conviene distinguir entre el capital humano y las características organizacionales.

Dentro del capital humano, se consideran características como la edad del emprendedor (Lasch y Yami, 2007), donde ofrecen una reseña de varios estudios que demuestran cómo los emprendedores innovadores son, en promedio, de dos a cinco años mayores que aquéllos no innovadores. No obstante, la edad está relacionada con el nivel educativo, cuyo efecto tiene un impacto positivo en la innovación. Levenburg y Kosalge (2006) demostraron que la educación de quienes dirigen la empresa es un determinante positivo en la adopción de innovaciones tecnológicas. La educación aumenta la complejidad cognitiva, permitiéndole al emprendedor tener una visión más amplia y abierta hacia la innovación. Por tanto, un mayor nivel educativo está relacionado con una mayor capacidad de innovación.

Dado que las empresas innovadoras se caracterizan por tener líderes dispuestos a asumir riesgos (Khan y Manopichetwattana, 1989), se puede pensar que la motivación, autonomía y autoconfianza del emprendedor influyen positivamente en la capacidad para innovar de la empresa.

Dentro de estas características organizacionales se encuentra la inversión inicial. Según Lasch et al. (2007), la disponibilidad de un fuerte capital inicial incide en el crecimiento de las empresas tecnológicas, donde la innovación es fundamental. En cierta forma, una mayor inversión inicial produce un impacto positivo en la capacidad de innovación, debido a que concede solvencia económica a la empresa para empezar sus actividades con un tamaño óptimo, evitando así obstáculos que puedan asfixiar la innovación.

La edad de la empresa se considera otra característica importante. Goode y Stevens (2000) determinaron que las empresas consolidadas eran más propensas a innovar que las más jóvenes, debido a su experiencia y disponibilidad de recursos para adoptar innovaciones.

Las empresas también pueden ser más innovadoras compitiendo en mercados internacionales, donde la existencia de una mayor rivalidad hace que la innovación constituya una vía necesaria para sobrevivir. De hecho, la intensidad de exportación es mayor entre las empresas intensivas en I+D e innovación (Bleaney y Wakelin, 2002).

La composición del equipo emprendedor es otra característica organizacional que influye en la capacidad para innovar (Souitaris, 1999). Una parte de la teoría sostiene que el éxito en la toma de decisiones aumenta cuando el emprendedor es apoyado por un equipo de socios fundadores (Schutjens y Wever, 2000). Las ventajas de dicho apoyo radican en la complementariedad de los socios, la disponibilidad de un mayor stock de conocimiento y la división de las tareas (Lasch et al., 2007).

El tamaño de la empresa se ha asociado al éxito de la misma (Geroski, 1995; Agarwal y Audretsch, 2001). Las grandes empresas tienen mayores recursos, habilidades, experiencia y probabilidades de supervivencia que las pequeñas.

Dentro de los determinantes externos, se puede distinguir, la dimensión del entorno 
vinculada a la actividad económica y la dimensión del entorno relacionada con el área geográfica en el que se localiza el negocio.

Noronha et al. (2006) sostienen que las condiciones del entorno pueden afectar a la capacidad de innovación de las pequeñas empresas. El entorno en el que nacen las nuevas empresas, desempeña un papel clave a la hora de definir y determinar el comportamiento estratégico que vayan a desarrollar éstas durante su etapa de infancia.

Dentro del proceso de innovación de un sector industrial, a menudo se diferencia la fase exploratoria de la fase de explotación de la innovación (Acs y Audretsch, 1990). Determinados sectores industriales se encuentran en una fase incipiente dentro del ciclo de vida del sector, lo que hace suponer que el esfuerzo en innovación sea principalmente de carácter exploratorio. Es decir, se invierte mucho en $\mathrm{I}+\mathrm{D}+\mathrm{i}$ con el fin de que en un futuro, más o menos cercano, se pueda desarrollar y comercializar exitosamente un nuevo producto que por lo menos rentabilice la inversión realizada durante la fase de $\mathrm{I}+\mathrm{D}+\mathrm{i}$.

Las características propias del enclave geográfico en el que se ubica la nueva organización, es otro factor que se debe considerar. No todas las regiones tienen la misma capacidad de innovación. Existe una variabilidad patente de la actividad de innovación entre distintas regiones. Por ello, se considera necesario añadir el factor de territorialidad en el análisis del efecto que ejerce el entorno en la propensión de innovar de una organización.

El Manual de Bogotá, propone un set de indicadores a tener en cuenta en el proceso de medición de la capacidad y proceso de innovación, dentro de los cuales se encuentran: identificación de la firma, desempeño económico de la empresa, actividades de innovación (indicadores de gasto y de frecuencia), resultados de la innovación, objetivos de la innovación, fuentes de información para la innovación, financiamiento de la innovación, relación con el sistema nacional de innovación, factores que afectan la innovación, y evaluación de políticas gubernamentales en materia de innovación, ciencia y tecnología y competitividad.

Considerando la importancia que el sector cerámico representa para el Norte de Santander (Comisión Regional de Competitividad Norte de Santander, 2010) y las manifestaciones de apoyo que se han brindado por medio de la Gobernación de Norte de Santander, la Universidad Francisco de Paula Santander, el Consejo Departamental de la Ciencia y Tecnología de Norte de Santander, y Colciencias, a través de diferentes proyectos tendientes a la conformación e implementación del Clúster Cerámico, se justifica el desarrollo de esta investigación porque proporciona información suficiente para la comprensión de los procesos de innovación tecnológica en el contexto local, los factores que los afectan y sus impactos, los cuales servirán como insumo estratégico para los tomadores de decisiones en el ámbito académico, empresarial y político, sobre el fomento de la innovación tecnológica, factor importante para generar una ventaja competitiva.

\section{Materiales y métodos}

La población de la investigación, está conformada por 59 empresas cerámicas, registradas ante la Cámara de Comercio de Cúcuta a fecha de junio de 2007, las cuales se encuentran ubicadas en los municipios de Cúcuta, el Zulia, Los Patios, Villa del Rosario y San Cayetano. La muestra se determinó apoyándose en la fórmula de Weiers (1986, p.123), la cual fue de 37 empresas. La investigación se desarrolló en las etapas que se exponen a continuación.

Etapa 1. Inicialmente, se definieron los indicadores a tener en cuenta para determinar el perfil de la empresa, la capacidad de innovación tecnológica de la empresa y el 
proceso de innovación, apoyándose en el marco teórico anteriormente expuesto. Estos indicadores fueron validados por un experto en el área de gestión de tecnología y un experto del sector cerámico.

Etapa 2. Con relación a estos indicadores, con el objeto de realizar la caracterización del proceso de innovación en las empresas cerámicas del sector, se diseñó un cuestionario de 49 preguntas, las cuales fueron de diferentes tipo: dicotómicas, selección con única respuesta, selección múltiple, escala likert y preguntas abiertas.

Etapa 3. Se realizó la validación de contenido, por medio de revisión de expertos, donde se consultó a tres (3) investigadores de las áreas de estadística, metodología de la investigación y en gestión de tecnología.

Etapa 4. Posteriormente se realizó una prueba piloto a 10 empresas, donde se observó que las preguntas fueron contestadas por todas la empresas y no se presentó inconvenientes en su comprensión, y el tiempo promedio de diligenciamiento fue de 25 minutos.

Etapa 5. Se determinó la confiabilidad del cuestionario en las preguntas tipo escala, utilizando el modelo expuesto por Cronbach (1951), donde se obtuvo un nivel de confiabilidad alto, en las diferentes escalas que fue de $0,81,0,89$ y 0,83.

Etapa 6. Los datos recolectados se ordenaron, procesaron y analizaron utilizando la estadística descriptiva, de Microsoft Office (Excel).

Etapa 7. Los resultados del proceso de medición de la capacidad de innovación tecnológica y el proceso de la innovación, se sintetizaron a través de perfiles, donde identificó el nivel de contribución de cada dimensión analizada para estas variables, utilizando la siguiente escala: nulo ( 0 al $5 \%$ ), muy bajo (6 al 40\%), bajo (41 al $70 \%$ ), alto (71 al 90\%), muy alto (91 al 100\%), y se clasificó cada indicador dependiendo de los resultados favorables obtenidos. Los resultados obtenidos en esta fase, se presenta en el siguiente numeral.

Etapa 8. Corresponde a las conclusiones del estudio, las cuales se presentan al final del presente artículo.

\section{Resultados y discusión}

\section{Capacidad de innovación tecnológica.} A partir de la información analizada en cada una de las dimensiones de la capacidad de innovación, se sintetizó a través de una escala de 5 niveles, que permite medir la contribución de cada indicador a la capacidad de innovación tecnológica.

Figura 1. Nivel de contribución de la dimensión de capital humano a la capacidad de innovación tecnológica de las empresas del sector cerámico del área metropolitana de Cúcuta

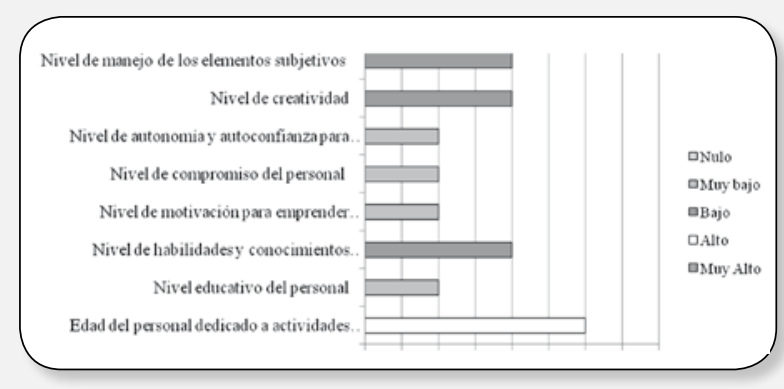

Se puede observar en la figura 1 , que el capital humano representa una debilidad para la capacidad de innovación de las empresas, ya que su personal no cuenta con el conocimiento técnico, la motivación y emprenderismo necesario para asumir riesgos y afrontar el desarrollo de actividades de $\mathrm{I}+\mathrm{D}+\mathrm{I}$. De acuerdo a los datos obtenidos se pudo observar que, en las empresas que han desarrollado algún tipo de innovación en producto o proceso, el personal encargado de estas actividades se caracteriza en su mayor parte por tener una edad entre 30 y 40 años con un $38 \%$, y entre 40 y 50 años con un $31 \%$, lo cual evidencia la importancia de la experiencia y conocimiento del proceso para emprender este tipo de actividades, lo cual coincide con lo expuesto por Lasch et al., (2007) quienes 
señalan que los emprendedores innovadores son, en promedio, mayores que aquellos no innovadores. Con respecto al nivel educativo, se observa que en las empresas del sector cerámico, la mayor parte del personal a nivel administrativo posee estudios profesionales, seguidos de los estudios de secundaria, y una mínima cantidad posee estudios a nivel especialistas y magister. A nivel de producción es muy poco el personal con estudio de pregrado, predominando la presencia de personal con estudio de primaria y secundaria.

De acuerdo a lo expresado por Levenburg et al. (2006) el nivel de formación es un factor importante para la adopción tecnológica en las industrias, por lo cual se puede inferir que el bajo nivel de formación que tiene el personal de las empresas del sector cerámico limita su capacidad de innovación. Por otra parte, existe una baja cultura organizacional orientada hacia la innovación, donde los empleados se limitan únicamente a realizar su trabajo rutinario sin hacer ningún aporte que contribuya a la mejora de los procesos o al desarrollo de nuevos productos. Esta situación no favorece la capacidad de innovación de las empresas, de acuerdo a lo expuesto por Khan y Manopichetwattana (1989), quienes manifiestan que las empresas innovadoras se caracterizan por tener líderes dispuestos asumir riesgos.

Figura 2. Nivel de contribución de la dimensión de los aspectos organizacionales a la capacidad de innovación tecnológica de las empresas del sector cerámico del área metropolitana de Cúcuta

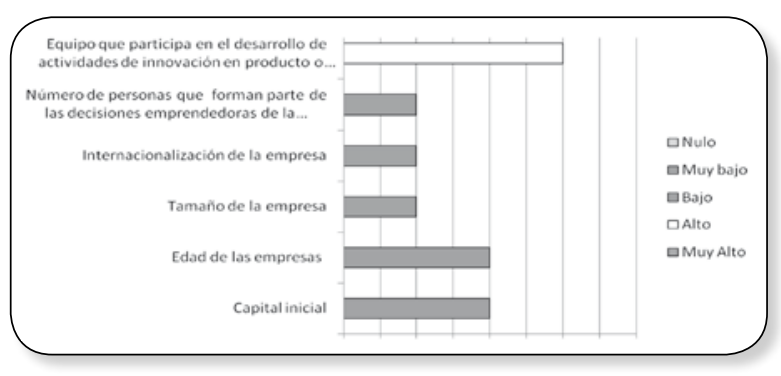

En la figura 2, se muestra que en las empresas existe un nivel de contribución muy bajo para la capacidad de innovación, en relación al tamaño de la empresa, su actividad de internacionalización y el número de personas que forman parte de las decisiones emprendedoras; en un nivel bajo se encuentra la edad de las empresas y el capital inicial; y en un nivel alto se encuentra el equipo que participa en el desarrollo de las actividades de innovación. Se puede deducir que el componente organizacional de las empresas se encuentra poco sólido y favorable para la actividad de innovación, de acuerdo a lo expuesto por los siguientes autores: Lasch et al. (2007), la disponibilidad de un fuerte capital inicial incide en el crecimiento de las empresas tecnológicas, donde la innovación es fundamental; Goode y Stevens (2000), señalan que las empresas consolidadas son más propensas a innovar, debido a su experiencia y disponibilidad de recursos para adoptar innovaciones, por lo que la condición de tener empresas tan jóvenes en el sector cerámico, no favorece la actividad innovadora; Geroski (1995) y Agarwal y Audretsch (2001), exponen que las grandes empresas tienen mayores recursos, habilidades, experiencia y probabilidades de supervivencia que las pequeñas; Bleaney y Wakelin (2002), afirman que las empresas son más innovadoras compitiendo en mercados internacionales; y Schutjens y Wever (2000), expresan que el emprendedor debe ser apoyado por un equipo complementario donde exista un mayor stock de conocimiento.

En la figura 3, se puede observar, en forma general, que la mayor parte de las empresas presentan un nivel financiero medio - bajo, y solo un porcentaje pequeño presenta un nivel financiero alto. El nivel financiero de las empresas del sector cerámico, no favorece la capacidad de innovación ya que no existe la suficiente solvencia económica que permita a las empresas invertir constantemente en actividades de investigación y desarrollo tecnológico para obtener resultados a mediano o largo plazo. 
Figura 3. Nivel financiero de las empresas

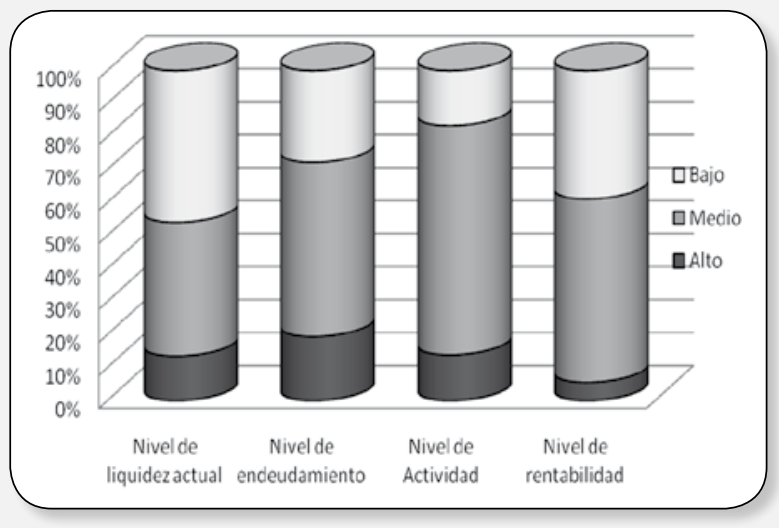

En la figura 4, se muestra que en las empresas existe un comportamiento muy bajo de exploración de las actividades de $\mathrm{I}+\mathrm{D}$, lo cual limita su nivel tecnológico y capacidad de aprendizaje para lograr avanzar y obtener innovaciones de mayor valor agregado producto de la investigación y desarrollo, y no de la imitación y adaptación.

Figura 4. Nivel de contribución de la dimensión del comportamiento de la exploración de $\mathrm{I}+\mathrm{D}$ a la capacidad de innovación tecnológica de las empresas del sector cerámico del área metropolitana de Cúcuta.

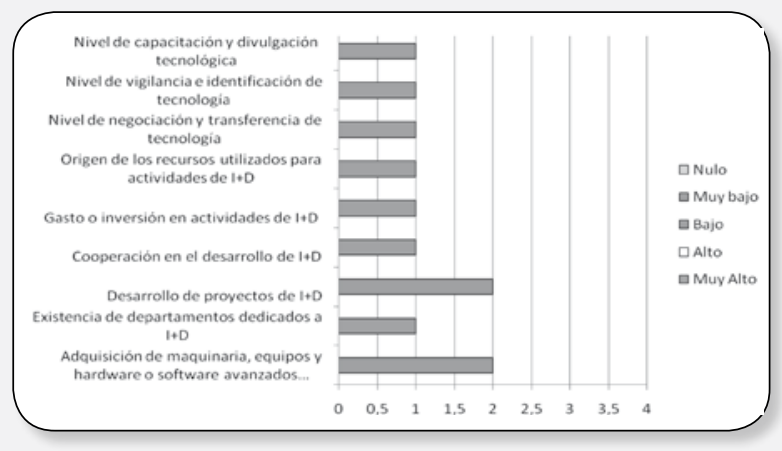

Figura 5. Número y tipo de protección que han adquirido las empresas para sus innovaciones tecnológicas en los últimos 5 años

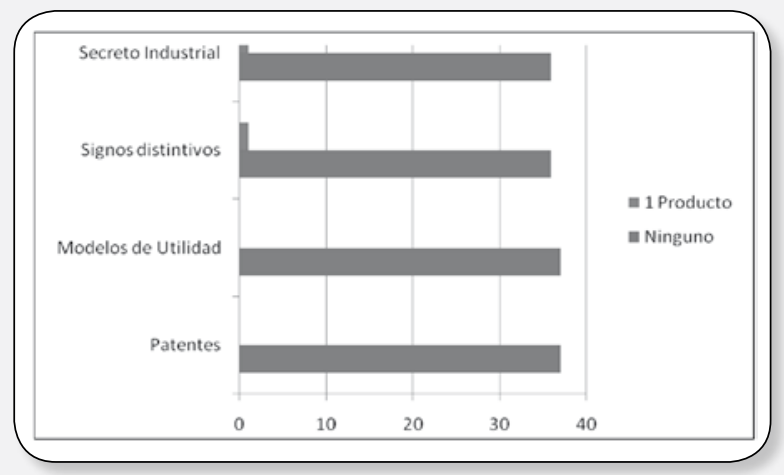

En relación a la actividad de explotación, se puede observar en la figura 5 que es nula la protección que se le ha dado a las innovaciones tecnológicas realizadas en las empresas, ya que su nivel es de tipo imitativo e incremental, lo cual no representa un grado novedoso que permita su protección a través de una patente o modelo de utilidad. Esta situación limita su nivel tecnológico y capacidad de aprendizaje para lograr avanzar y obtener innovaciones de mayor valor agregado producto de la investigación y desarrollo, de acuerdo a lo expresado por Acs y Audretsch (1990). Por lo tanto, hasta que no exista un nivel adecuado de exploración de $\mathrm{I}+\mathrm{D}$ no se podrá obtener productos que se puedan proteger para ser explotados y comercializados.

\section{Procesos de innovación}

Se sintetizó la información analizada en cada uno de los indicadores que se utilizaron para determinar el perfil de la innovación, a través de la escala de 5 niveles, donde se midió la contribución de cada indicador al procesos de innovación.

Figura 6. Grado de desarrollo alcanzado por las innovación tecnológica en las empresas del sector cerámico del área metropolitana de Cúcuta

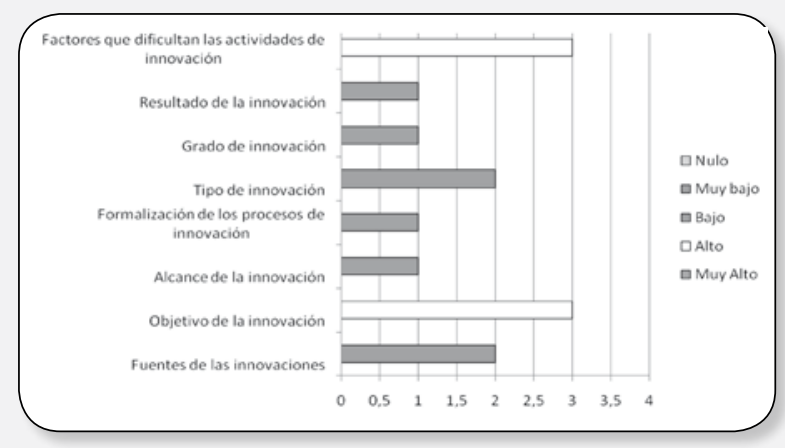

En la figura 6, se refleja que el perfil de las innovaciones tecnológicas llevadas a cabo en las empresas del sector cerámico, presentan un grado de desarrollo muy bajo en cuanto al alcance de las innovaciones, la formalización del proceso de innovación, el grado de innovación y los resultados alcanzados; en un grado de desarrollo bajo se encuentran las fuentes de las innovaciones y el tipo de 
innovación desarrollada, y en un grado alto se encuentra los objetivos que persiguen los procesos de innovación. Con respecto a los factores que dificultan la innovación tecnológicase consideranque estosrepresentan un nivel alto de obstáculo en el desarrollo del proceso de innovación. Se puede deducir que el perfil de las innovaciones alcanzadas por las empresas del sector cerámico representan un bajo grado de desarrollo, básicamente por la informalidad de los procesos de innovación, los cuales no son fruto de las actividades de I + D, el carácter adaptativo e incremental de los desarrollos exógenos, la mínima articulación de las empresas con el entorno, el bajo capital humano competente para la innovación, la escaza cantidad de recursos asignados a infraestructura y desarrollo de las actividades de $\mathrm{I}+\mathrm{D}$.

Dentro de los factores humanos que las empresas consideran que obstaculizan las actividades de innovación se encuentran la falta de personal calificado con una participación del $44 \%$, resistencia al cambio en un $31 \%$, falta de experiencia del personal con un $19 \%$, y reducción del empleo en un $6 \%$. Estos resultados ratifican el bajo nivel de formación que tienen los empleados donde no existen personas a nivel de producción que posean estudios de postgrado de maestrías y doctorados, que permitan aumentar el dinamismo de los procesos de investigación y desarrollo tecnológico.

De igual forma la resistencia al cambio es un factor obstaculizador, ya que más del 60\% no ha realizado ningún proceso de innovación y ha continuado con sus productos y procesos tradicionales. Dentro de los factores económicos, se encuentran la dificultad de obtener financiamiento con un $43 \%$, el riesgo económico con un $37 \%$, riesgo técnico y periodo de retorno muy largo con un $8 \%$ cada uno y difícil control de gastos de innovación con un $4 \%$. A pesar de las diferentes fuentes de financiación que existen en el país para promover la innovación y competitividad de las mipymes las empresas consideran que los procesos y requisitos para acceder a los recursos son muy complejos, y que no cuentan con la experiencia, personal capacitado y la contrapartida necesaria.

Con base a los hallazgos encontrados en los proceso de innovación realizados en las empresas cerámicas del área metropolitana de Cúcuta, se puede inferir que siguen la misma tendencia de los resultados obtenidos en la industria colombiana (Malaver y Vargas, 2004), donde la mayoría de las empresas se caracterizan por tener procesos informales de innovación los cuales son emergentes y buscan atender básicamente la demandas de los clientes, sus capacidades tecnológicas se construyen a nivel intermedio a través de la explotación, adaptación y desarrollo de las tecnologías incorporadas y las innovaciones en producto son las que más predominan en las empresas.

Figura 7. Nivel de impacto económico observado con las innovaciones tecnológicas en producto o proceso

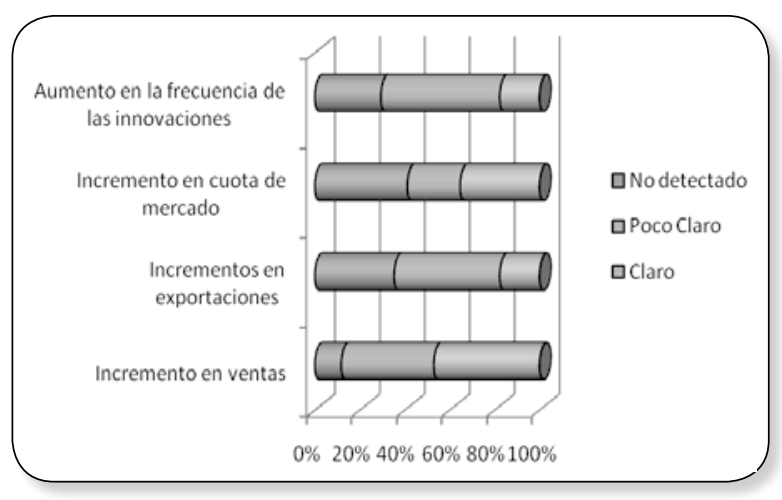

En la figura 7, se observa que el impacto de las innovaciones tecnológicas en productos $\mathrm{o}$ procesos, que las empresas del sector cerámico han evidenciado de forma más clara, es el incremento en las ventas; seguido en menor medida del incremento de la cuota de mercado y de forma poco clara del incremento de las exportaciones y del aumento en la frecuencia de las innovaciones. Estos resultados están relacionados con el objetivo expuesto en los procesos de innovación de productos los cuales son la apertura de nuevos mercados y la ampliación de la gama de productos. 
Actores que intervienen en los procesos de innovación tecnológica de las empresas cerámicas del área metropolitana de Cúcuta y tipo de aporte que realizan

Partiendo de la información analizada de las encuestas, se pudo detectar que:

- Las empresas poseen una débil articulación con otras empresas o instituciones científicas y tecnológicas para el desarrollo de actividades de I+D, donde solo el 30\% de ellas se relacionan para desarrollar este tipo de actividades.

- Las empresas que se relacionan para desarrollar actividades de I $+\mathrm{D}$, lo hacen en mayor proporción con la universidad, los grupos de investigación y los proveedores, seguido en menor proporción con la Corporación Autónoma Regional de Norte de Santander Corponor y los Centros de Investigación.

- El tipo de relación que más predomina entre las empresas del sector cerámico con las otras entidades es la solicitud de información, seguida de la capacitación y la elaboración de ensayos.

- Las instituciones o entidades con las que se relacionan con mayor frecuencia son: la Asociación de Industriales de la Arcilla de Norte de Santander (Induarcillas), Centro de Investigación de Materiales Cerámicos (CIMAC), Universidad, Corporación Autónoma Regional de Norte de Santander (Corponor), Cámara de Comercio de Cúcuta y Proexport Colombia.

- Existe una debil articulación de las empresas del sector cerámico con el Sistema Nacional de Ciencia, Tecnología e Innovación.

\section{Conclusiones}

Se estableció el análisis interno de la innovación tecnológica del sector cerámico del área metropolitana de Cúcuta, lográndose identificar que la mayoría de ellas por su condición de micro, pequeñas y medianas empresas se caracterizan por:

1. Tener debilidad en los aspectos administrativos, organizacionales y tecnológicos.

2. Presentar un bajo nivel en su capital humano, el cual no cuenta con la formación profesional, motivación, autonomía, autoconfianza, creatividad y compromiso para asumir retos y potencializar las actividades de $\mathrm{I}+\mathrm{D}$.

3. Tener poca solidez en los aspectos organizacionales, los cuales no favorecen las actividades de innovación, por el bajo tamaño y edad de las empresas, la poca actividad de exportación, el bajo capital inicial y el escaso número de personas que forman parte de las decisiones emprendedoras.

4. Poseer un nivel financiero poco favorable para la innovación, por no contar con la suficiente solvencia económica para invertir constantemente en actividades de investigación y desarrollo tecnológico.

5. Contar con un comportamiento muy bajo de exploración de las actividades de $\mathrm{I}+\mathrm{D}$, lo que afecta su nivel tecnológico y capacidad de aprendizaje para obtener innovaciones más sistemáticas y de mayor impacto.

6. Carecer de un adecuado nivel de protección del conocimiento, lo cual se deriva de su bajo nivel de exploración de $\mathrm{I}+\mathrm{D}$.

7. Tener procesos informales de innovación, donde sobresalen las innovaciones en productos las cuales se caracterizan por ser de tipo imitativo e incremental de los desarrollos exógenos y no como resultado de las actividades de $\mathrm{I}+\mathrm{D}$.

Solo el $30 \%$ de las empresas del sector 
La innovación tecnológica en las empresas del sector cerámico del área metropolitana de Cúcuta cerámico del área metropolitana de Cúcuta se relacionan con otras empresas o instituciones científicas y tecnológicas para el desarrollo de actividades de I+D. El tipo de relación que más predomina entre las empresas del sector cerámico son: la solicitud de información, la capacitación y la elaboración de ensayos.

Existe una débil articulación de las empresas del sector cerámico del área metropolitana de Cúcuta con el Sistema Nacional de Ciencia, Tecnología e Innovación, lo cual se debe más a la falta de relación que a problemas entre la relaciones de los actores.

Las empresas del sector cerámico del área metropolitana de Cúcuta, deben velar y solicitar que dentro de los Planes de Desarrollo Departamental y Municipal, queden establecidas las apuestas a los sectores productivos, se destinen recursos y prioricen proyectos que favorezcan la competitividad del sector.

Las empresas del sector cerámico del área metropolitana de Cúcuta, deben unirse a la Asociación de Industriales de la Arcilla, Induarcillas, para gestionar mancomunadamente recursos, capacitaciones y apoyo de las diferentes entidades estatales, gubernamentales y municipales que tienen como misión fortalecer los sectores industriales. De igual forma, debe lograrse un mayor acercamiento entre la academia y el sector productivo, donde los grupos desarrollen investigación aplicada que responda a la problemática del sector y a través de los estudiantes de pregrado y posgrado se fomente el desarrollo de trabajos de grado que permitan mejorar los procesos administrativos, de producción, de calidad y tecnológicos, de tal forma de que se vayan minimizando las debilidades y mejorando su posición competitiva.

Se deben emprender en las empresas del sector cerámico la construcción de una cultura innovadora que propicie el desarrollo de ambientes creativos en las diferentes áreas del proceso.

\section{Agradecimientos}

A la Universidad Francisco de Paula Santander y a las empresas del sector cerámico de Norte de Santander, quienes apoyaron el desarrollo de esta investigación.

\section{Referencias bibliográficas}

Acs, Z. J. y Audretsch, D. (1990). Innovation and Small Firms. Cambridge: MIT Press.

Agarwal, R. y Audretsch, D. (2001). Does Entry Size Matter?. The impact of the life cycle and technology on firm survival. The Journal of Industrial Economics, 49(1), pp. 21-43.

Bleaney, M. y Wakelin, K. (2002). Efficiency, Innovation and Exports. Oxford Bulleting of Economics and Statistics, 64(3), pp. 3-15.

Cronbach, L. J. (1951). Coefficient alpha and the internal structure test. Psycometrika, 16. Septiembre, p. 291-334.

Geroski, P. A. (1995). What Do We Know About Entry?. International Journal of Industrial Organization, 13(4), pp. 421-440.

Goode, s. y Stevens, K. (2000). An Analysis of the Business Characteristics of Adopters and Non-adopters of World Wide Web Technology. Information Technology and Management, 1(1-2), pp. 129-154.

Hadjimanolis, A. (2000). A Resourcebased View of Innovativeness in Small Firms. Technology Analysis \& Strategic Management, 12 (2), pp. 263-281.

Imai, M. (1989). Kaizen, la clave de la ventaja competitiva japonesa. CEC-S.A: México.

Jaramillo, H.; Lugones, G., y Salazar, M. (2000). Manual para la normalización de indicadores de innovación tecnológica 
en América Latina y el Caribe, Manual de Bogotá. Bogotá, OEA/RICyT, Tres Culturas.

Khan, A. M. y Manopichetwattana, V. (1989). Innovative and non-innovative small firms: types and characteristics. Management Science, 35, pp. 597-606.

Lasch, F.; Le Roy, F. y Yami, S. (2007). Critical Growth factos of ICT Start-Ups. Management Decision, 45(1), pp. 62-75.

Levenburg, N.; Magal, S. R. y Kosalge, P. (2006). An exploratory investigation of organizational factors and e-business motivations among smfoes in the US. Electronic Markets, 16(1), pp. 70-84.

Malaver, F. y Vargas, M. (2004). Hacia una caracterización de los procesos de innovación en la industria colombiana. Los resultados de un estudio de casos. Revista Latinoamericana de Administración, Cladea, Bogotá.

Mandonado, E., Fernández, J.F. y Doiro, M. (2003). La innovación tecnológica en las organizaciones. Madrid: Thompson.(pp. 20).

Noronha, M. T.; Cesário, M. y Fernandes, S. (2006). Interaction between innovation in small firms and their environments: an exploratory study. European Planning Studies, 14(1), pp. 95-117.

OCDE. (2005). Manual de Oslo Guía para la recogida e interpretación de datos sobre innovación. Tercera Edición. OCDEEUROSTAT. Paris-Bruselas. Documento Electrónico, disponible en http://www.tragsa. es/SiteCollection Documents/Relaciones\%20 Institucionales\%20(Prensa)/Publicaciones/ Manual_de_Oslo.pdf

Pineda Serna, Leonardo. (2008). Enfoques alrededor de la gestión estratégica de la innovación. Documento de trabajo del programa de formación de alto nivel en gestión estratégica de la innovación FANGEI del Centro de Gestión de Conocimiento e Innovación CGCI de la Universidad del
Rosario. Disponible en: http://ssrn.com/ abstract $=1316446$

Porter, M. (1990). The Competitive advantage of nations. Londres: Mac Millan.

Schutjens, V. y Wever, E. (2000). Determinants of new firm success. Papers of Regional Science, 79(2), pp. 135-153.

Soutaris, V. (1999). Research on the Determinants of Technological Innovation. A Contingency Aproach», International Journal of Innovation Management, 3(3), pp. 287-305.

Weiers, R. (1986). Investigación de Mercados. México: Ed. Prentice Hall. 